\title{
Control of Ionization Processes in High Band Gap Materials
}

\author{
Matthias WOLLENHAUPT ${ }^{* 1}$, Lars ENGLERT ${ }^{* 1}$, Alexander HORN ${ }^{* 1}$ and Thomas BAUMERT ${ }^{* 1}$ \\ ${ }^{* 1}$ Universität Kassel, Institut für Physik und CINSaT, Heinrich-Plett-Str.40, D-34132Kassel, \\ Germany \\ E-mail: wollenha@physik.uni-kassel.de
}

\begin{abstract}
An overview on femtosecond laser pulse shaping techniques applied to control of the initial photo-physical steps involved in materials processing is presented. First, pulse shaping methodology in frequency domain is introduced and examples of shaped pulses relevant to laser microfabrication are discussed. Then, the use of tailored femtosecond pulses to control the initial steps of laser processing of high band gap materials is demonstrated. In particular, control on basic ionization processes acting as the initial photo-physical step of the ablation dynamics is exerted by highly asymmetric femtosecond laser pulse shapes generated by Third Order Dispersion (TOD).
\end{abstract}

DOI: 10.2961/jlmn.2009.03.0001

Keywords: Pulse shaping, spectral modulation, femtosecond spectroscopy, photo-ionization, laser nanoprocessing

\section{Introduction}

Ultra-short laser radiation has emerged as a powerful tool for processing materials, e.g. dielectrics, ranging from industrial precision micro machining to life science demands [1-6]. Dielectrics are mainly transparent for visible and near infrared radiation, therefore material modifications, like refractive index changes, ablation, void creation and filamentation, are mainly evoked by nonlinear processes requiring large intensities available for example via ultra-short laser radiation. Recent experiments on laser ablation indicate a strong dependence of the ablation processes on the pulse duration [7-9]. The advance from investigations regarding the pulse duration to those accounting the temporal profile of the pulses has shown a strong influence on laser induced damage, demonstrated in double pulse experiments [10-13] and symmetric pulse sequence experiments [14].

Pulse shaping techniques make use of the enormous coherent bandwidth associated with ultra-short laser radiation in order to exert full control over the light field in terms of intensity profile, instantaneous frequency and state of polarization. In the recent decade, femtosecond pulse shaping has been successfully applied to coherent control of atomic and molecular dynamics with applications ranging from laser science, quantum optics, atomic and molecular physics, solid state physics, photochemistry to biophysics, or quantum computing [15-18]. Therefore, the use of pulse shaping technology will be the key to open up new prospects in advanced materials processing by adapting the temporal energy flux of the laser radiation to the dynamical processes of excitation (e. g. electron excitation, defect generation and trapping, and color center generation) and pulse propagation (self-focusing, plasma defocusing, and filamentation).

This contribution is subdivided in two parts: the first part introduces pulse shaping by spectral phase modulation elucidating general properties of phase modulation. Depending on the experimental conditions exemplary phase functions are presented and discussed. In the second part experimental results on laser surface damage of fused silica with temporally asymmetric shaped femtosecond laser pulses obtained by introducing third order dispersion
(TOD) in the applied radiation via spectral phase modulation [19] will be presented demonstrating the potential of micromachining with tailored ultrafast laser radiation.

\section{Pulse shaping via spectral phase modulation}

In this section a short overview on spectral pulse shaping techniques is given including the discussion of special pulse shapes relevant to laser materials processing. Among the different kinds of pulse shaping, methods based on manipulation of the spectral phase function $\varphi(\omega)$ are most common to femtosecond pulse shaping [20]. The concepts of spectral pulse shaping were recently reviewed in [21]. In short, the spectrum $\square(\omega)$ of the unmodulated pulse envelope $\square(t)$ is modulated in frequency domain by the spectral transfer function $\square(\omega)$ to deliver the modulated spectrum

$E_{\text {mod }}(\omega)=E(\omega) \cdot M(\omega)$

Phase modulation is described by the spectral phase function $\varphi(\omega)$, i.e.

$M(\omega)=e^{-i \varphi(\omega)}$

Recombining the spectral components produces the complex valued modulated temporal field envelope $\square_{\bmod }(\mathrm{t})$, which is decomposed into its modulus $\left|\square_{\text {ìेंx }}(\mathrm{t})\right|$ and the temporal phase $\zeta(t)$

$\mathrm{E}_{\text {mod }}(t)=\left|\mathrm{E}_{\text {mod }}(t)\right| e^{i \zeta(t)}$

Note that the physical field including the laser carrier oscillation at the frequency $\omega_{0}$ is the real part of the product of pulse envelope $\mathrm{E}_{\text {mod }}(t)$ and the carrier oscillation

$E_{\text {mod }}(t)=\mathrm{E}_{\text {mod }}(t) \cdot e^{i \omega_{0} t}$.

Changes of the instantaneous frequency $\Delta \omega(t)$ are described by the derivative of the temporal phase $\zeta(t)$, i.e.

$\Delta \omega(t)=\frac{d}{d t} \varsigma(t)$

\subsection{Experimental implementation}

An experimental implementation of a spectral phase modulator generating quasi-deliberate phase functions can be achieved by a 4f-setup usually applied in chirped pulse 
amplification for temporal stretching and compression of ultrafast radiation. Here, the ultrashort laser radiation is spatially resolved in its spectral components by a grating and imaged onto a liquid crystal display (LCD) situated in the Fourier plane of the $4 \mathrm{f}$-setup. In the Fourier plane the spectral phase shaping takes place by space-selective phase modulation of the LCD. After the LCD, the spectral components of the radiation are merged again by another grating. For highest possible throughput reflective optics are preferred. Especially the efficiency of gratings in terms of spectrally resolved reflectivity and/or transmittance of the laser radiation should be uniform over the laser spectrum. The LCD should feature a high damage threshold for optimal throughput and a large number of pixels allowing the production of complex shaped pulses ranging from the femtosecond to several tens of picoseconds.

\subsection{General properties of phase modulation}

One of the useful properties of phase modulation is the conservation of the laser energy, since the modulated Power Spectral Density (PSD)

$$
\operatorname{PSD}(\omega)=\left|\mathrm{E}_{\text {mod }}(\omega)\right|^{2}=\left|\mathrm{E}(\omega) \cdot e^{-i \varphi(\omega)}\right|^{2}=|\mathrm{E}(\omega)|^{2}
$$

is not affected by phase modulation. By virtue of Parseval's theorem this applies also to the modulated field in time domain, i.e.

$$
\int\left|\mathrm{E}_{\text {nod }}(t)\right|^{2} d t=\int|\mathrm{E}(t)|^{2} d t \text {. }
$$

For quantitative comparison of experimental results obtained by modulated pulses with those obtained using unmodulated pulses, Eqns. (6) and (7) imply that the sample is exposed to the same pulse energy and the same spectrum - the only difference being the temporal distribution of the laser radiation. In the case of amplitude modulation it is not a priori clear whether the observed processes induced by the laser radiation on a target are due to changes of the energy, the spectrum or the pulse shape.

One way to unambiguously reveal control exerted by the temporal structure of the modulated pulse is to study the effect of a time reversed replica of the modulated pulse. Using phase modulation techniques, time reversal is readily accomplished by spectral phase conjugation, i.e. $\varphi(\omega) \rightarrow-\varphi(\omega)$. This feature will be exploited in our experimental studies discussed in section 3 to disentangle two basic ionization processes.

In many cases it is advantageous to separate the influence of the temporal structure of the pulse envelope from effects due to the changes in the instantaneous frequency, i.e. changes in the photon energy. By using anti-symmetric phase functions $\varphi(-\omega)=-\varphi(\omega)$ the temporal envelope is always real valued implying constant instantaneous frequency $\omega(\mathrm{t})$. In our experimental studies (see section 3 ) an anti-symmetric phase function is used to assure constancy of the instantaneous frequency.

\subsection{Polynomial phase functions}

A simple approach to understand the physical significance of a spectral phase function $\varphi(\omega)$ with respect to the temporal pulse shape is based on its Taylor expansion resulting in a sum of polynomial phase functions

$$
\varphi(\omega)=\phi_{0}+\phi_{1} \cdot \omega+\frac{\phi_{2}}{2 !} \omega^{2}+\frac{\phi_{3}}{3 !} \omega^{3}+\ldots .
$$

In this section the effects due to the individual terms of the Taylor series on the temporal pulse shape is analyzed. The absolute phase, which relates the carrier oscillation to the envelope, is modulated if the first term $\phi_{0}$ is non-zero. Although this type of modulation can be important for coherent control experiments [22], it does not influence the pulse envelope and is therefore not considered here. In accordance with the Fourier shift theorem, the linear term in the spectral modulation function $\phi_{1} \omega$ is responsible for a time shift of the pulse envelope by $t=\phi_{1}$. Linear spectral modulation results in a time delay of the modulated pulse with respect to the unmodulated pulse, however it should be noted that, unlike using an interferometric set-up, the envelope is shifted rather than a copy of the pulse. The shifting properties of a linear phase function will be used in section 2.4 in order to interpret the effect of V-type spectral phase functions (see below).
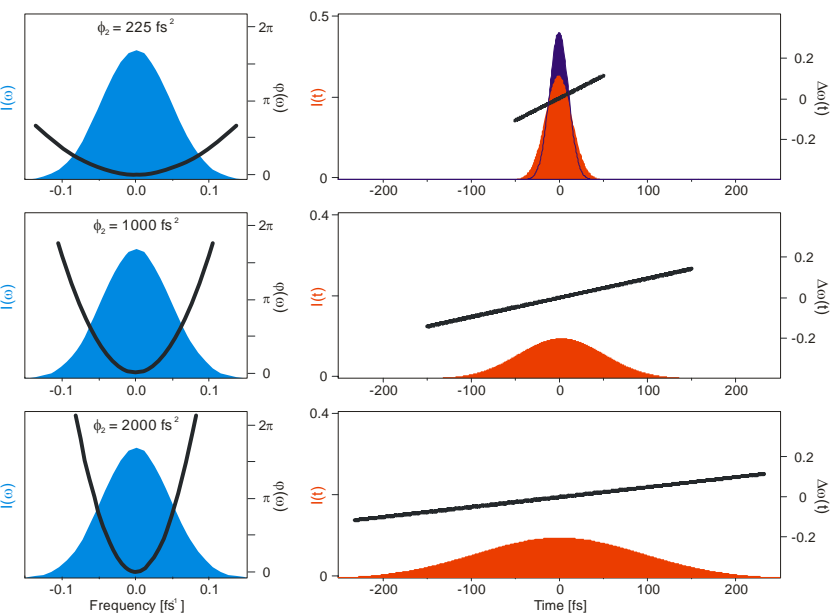

Fig. 1 Left: Quadratic spectral phase (GDD) with $\varphi(\omega)=\phi_{2} / 2 ! \omega^{2}$ for increasing chirp parameters $\phi_{2}$ applied to the spectrum of a Gaussian input pulse (blue) of $\Delta t=25 \mathrm{fs}$. Right: temporal envelope of the modulated pulse (red) and change of the instantaneous frequency $\Delta \omega(\mathrm{t})$. The chirp parameter $\phi_{2}$ increases from $225 \mathrm{fs}^{2}$ to $2000 \mathrm{fs}^{2}$. For comparison, the unmodulated pulse (blue in the background) is depicted in the upper panel.

Quadratic phase modulation - also termed Group Delay Dispersion GDD - using the spectral phase function

$$
\varphi(\omega)=\frac{\phi_{2}}{2 !} \cdot \omega^{2} \equiv \frac{\mathrm{GDD}}{2 !} \cdot \omega^{2}
$$

plays a major role in many applications based on femtosecond pulse manipulation techniques [23]. By applying GDD, the pulse duration of the laser radiation $\Delta \mathrm{t}_{\text {mod }}$ is modified and a linear frequency sweep is introduced. Assuming a bandwidth limited Gaussian input pulse with a pulse duration $\Delta \mathrm{t}$ (FullWidth at Half Maximum FWHM) the chirped pulse remains Gaussian shaped featuring an increased pulse duration of

$$
\Delta t_{\text {mod }}=\sqrt{\Delta t^{2}+\ln ^{2}(16)\left(\frac{\phi_{2}}{\Delta t}\right)^{2}}
$$

The frequency sweep is characterized by a linear change of the instantaneous frequency $\Delta \omega(\mathrm{t})=2 \alpha \mathrm{t}$, where 
$\alpha=\left\{2 \phi_{2}+\frac{\Delta t^{4}}{8 \phi_{2} \ln ^{2}(2)}\right\}^{-1}$

describes the chirp rate. A positive value of $\phi_{2}$ leads to an "up-chirp" characterized by an increase of the instantaneous frequency, whereas a negative $\phi_{2}$ implies a decrease of $\Delta \omega(\mathrm{t})$, known as "down-chirp". Fig. 1 shows the effect of quadratic spectral phase modulation to a Gaussian input pulse with a duration of $\Delta t=25 \mathrm{fs}$. With increasing chirp parameter $\phi_{2}$, the pulse duration increases according to Eq. 10. accompanied by the reduction in intensity. Note that, in accordance with Eq. (11), the chirp rate is a nonmonotonic function of the chirp parameter $\phi_{2}$. The maximum obtainable chirp parameter $\alpha$ for a given pulse duration $\Delta \mathrm{t}$ is obtained for

$$
\phi_{2}=\frac{\Delta t^{2}}{4 \ln (2)}
$$
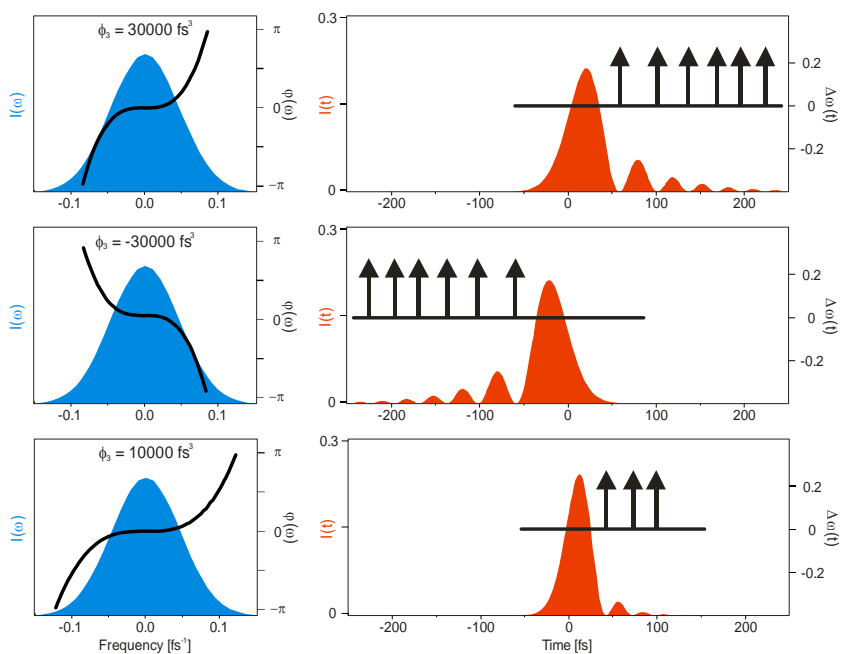

Fig. 2 Left: Third Order Dispersion (TOD) spectral phase functions $\varphi(\omega)=\phi_{3} / 3$ ! $\omega^{3}$ for positive and negative chirp parameters $\phi_{3}$. The arrows indicate delta functions of strength $\pi$, corresponding to a jump of the temporal phase by $\pi$, indicating a change of sign in the temporal envelope.

Third Order Dispersion (TOD) given by the spectral phase function

$$
\varphi(\omega)=\frac{\phi_{3}}{3 !} \cdot \omega^{3} \equiv \frac{\mathrm{TOD}}{3 !} \cdot \omega^{3}
$$

results in asymmetric pulse shapes analytically described by a damped Airy function [21,24]. Fig. 2 shows examples for TOD spectral phase modulation for positive and negative values of the parameter $\phi_{3}$ as well as a variation of the absolute value of $\phi_{3}$. The pulse shape is characterized by an intense initial pulse followed by a pulse sequence with deccying amplitudes. At the zeros of the damped Airy function the temporal phase jumps by $\pi$ leading to the (immaterial) delta discontinuities in the instantaneous frequency. Applying the anti-symmetric phase function of TOD results in a constant instantaneous frequency. The sign of $\phi_{3}$ controls the temporal direction of the pulse shape: positive values of $\phi_{3}$ lead to a series of post-pulses as shown in the upper panel of Fig. 2 whereas negative values of $\phi_{3}$ result in a series of pre-pulses. For these highly asymmetric pulses, the FWHM - which is generally employed to characterize simple bell-shaped distributions - is not a representative quantity to characterize the pulse duration. Instead, the statistical definition of the pulse duration yields a formula similar to Eq. (10) to describe the duration of pulse subjected to TOD

$$
\Delta t_{\bmod }=\sqrt{\Delta t^{2}+24 \ln ^{3}(2)\left(\frac{\phi_{3}}{\Delta t^{2}}\right)^{2}} .
$$

Note that in Eq. (14) the pre-factors are so chosen as to reproduce the value of $\Delta t$ obtained by taking the FWHM for an unmodulated pulse. With respect to materials processing the remarkable features of TOD are (i) temporal symmetry-breaking of the envelope implying control on the time-dependent energy flux onto the sample and (ii) the ability to produce a short intense pulse accompanied by a weak long pulse train. In Section 3 we will use this type of modulation to control basic ionization processes to manipulate the ablation dynamics.

\subsection{Pulse sequences}

Pulse sequences are a common tool to study dynamical properties of light matter interactions on the time scale of the pulses involved. By applying pulse sequences in coherent control, also phase sensitive effects similar to Ramsey fringes have been reported $[25,26]$. The use of pulse sequences in advanced applications to ultrafast material processing has been reported in [14].

Using an interferometric set-up is conceptually the simplest way to produce a sequence of pulses. However, practical considerations, such as the stability of an interferometer and alignment issues, but most importantly the unavoidable spectral modulations introduced by the interferometer, suggest alternative approaches based on pulse shaping techniques. Three phase functions to deliver pulse sequences, i.e. periodic functions, phase jumps and V-shaped phase functions [27] shall be discussed in this section.
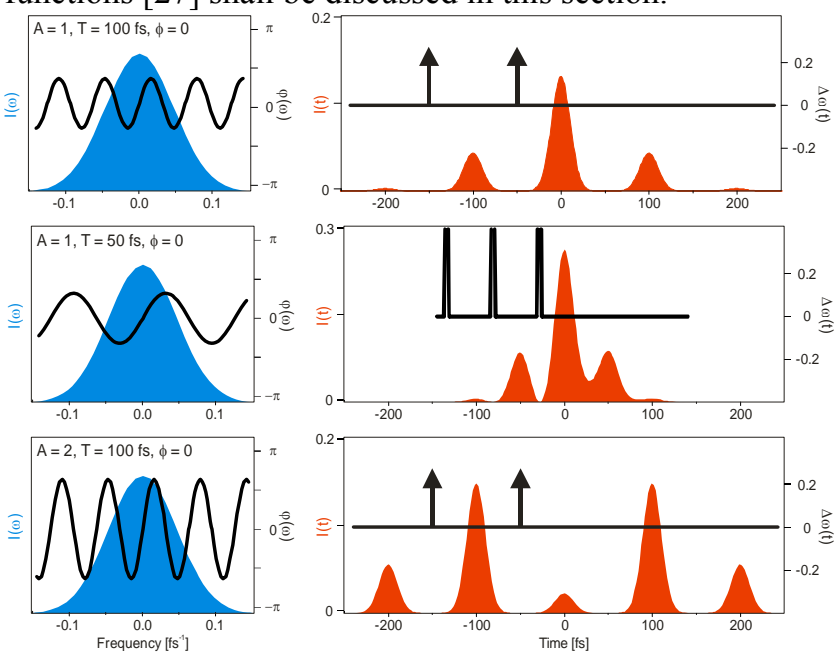

Fig. 3 Upper panel: sinusoidal spectral phase modulation with $A=1 ; T=100 \mathrm{fs}, \phi=0$. Middle: lowering the modulation frequency to $T=50 \mathrm{fs}$ merges the sub-pulses. Increasing the amplitude A results in an increased number of sub-pulses. The arrows indicate delta functions of strength $\pi$, indicating a change of sign in the temporal envelope of the field. 
Periodic spectral phase functions applied to modulate the spectrum of an ultrafast laser pulse deliver pulse sequences of controllable intensity, phase and temporal separation. It has been shown [21,27-29] that sinusoidal spectral phase modulation of the form

$$
\varphi(\omega)=A \sin (\omega T+\phi)
$$

produces a sequence of sub-pulses with a temporal separation determined by the parameter $T$ and controllable relative temporal phases determined by the absolute phase $\phi$

$$
\mathrm{E}_{\text {mod }}(t)=\sum_{n=-\infty}^{\infty} J_{n}(A) \mathrm{E}(t-n T) e^{-i n \phi}
$$

The amplitudes of the $n$th sub-pulse is given by $J_{n}(A)$, where $J_{n}$ describes the Bessel function of the first kind and order $n$, and is controlled by the modulation parameter $A$. Provided the individual sub-pulses are temporally separated, i.e. $T$ is chosen to exceed the pulse duration $\Delta t$, the envelope of each sub-pulse is a (scaled) replica of the unmodulated pulse envelope (Fig. 3 upper and lower panel). The (immaterial) delta functions of strength $\pi$ between the subpulses indicate again a change-of-sign in the pulse envelope. For smaller delay times $T$ the sub-pulses interfere with each other to deliver more highly structured pulse shaped (Fig. 3 middle panel), with real excursions of the instantaneous frequency.

So far, continuous phase functions have been considered. Now, a second class of spectral modulation functions modulation based on discontinuous functions is considered. It was realized [21,27], that spectral phase jumps

$$
\varphi(\omega)=\frac{\theta}{2} \sigma(\omega)
$$

where $\sigma$ denotes the signum function, deliver another method to produce a sequence of two "picosecond" pulses.
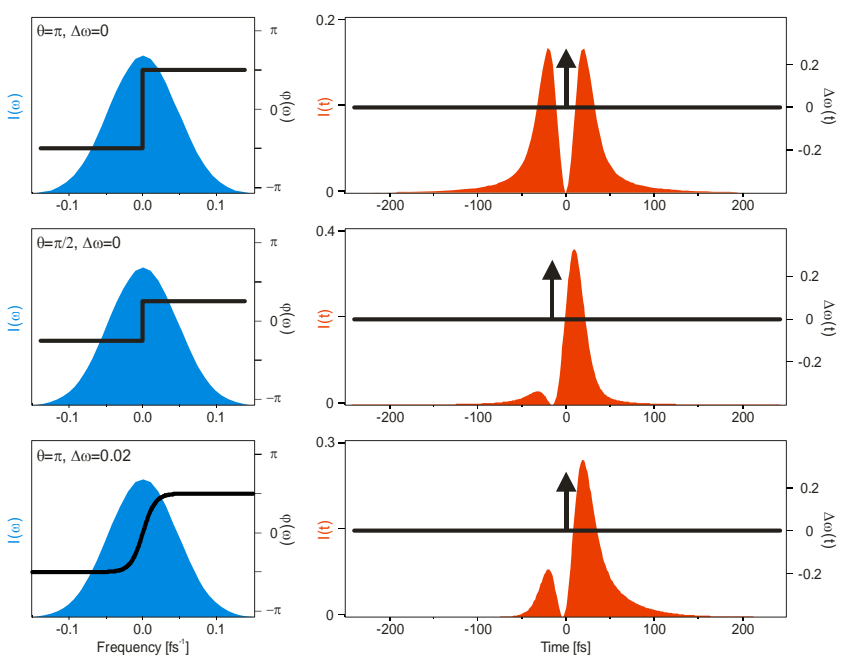

Fig. 4 Spectral phase function with a $\theta$-step at the central frequency. Upper panel: $\theta=\pi$ leads to symmetric picosecond double pulses separated approximately by $\Delta t$, middle: $\theta=\pi / 2$ results in a weak pre-pulse and an intense post-pulse, lower trace: blurring the $\pi$-step reduces the pulse duration.

Pulses resulting from spectral phase jumps have been used to manipulate coherent atomic dynamics [22,30-32]. An example for a discontinuity of $\pi$ at the central frequency in the spectral phase function is shown in the upper panel of
Fig. 4. This so-called $\pi$-step results in two pulses with larger duration delayed with respect to each other by approximately $\Delta t$. A slight generalization of this spectral phase is introduced by variation of the step-height. A phase jump of $\theta=\pi / 2$ breaks the temporal symmetry and is associated with a weak pre-pulse and an intense post-pulse (middle panel of Fig. 4). Due to this structure, generalized $\theta$ jumps might be suitable candidates for materials processing similar to TOD. Blurring the phase discontinuity is an alternative approach to deliver asymmetric pulses. In addition, blurred phase functions deliver much shorter pulses a property which is required to manipulate ultrafast dynamics on the sub-picosecond level.

Finally, we introduce the V-type phase function

$$
\varphi(\omega)=\tau|\omega-\delta \omega|
$$

shown in Fig. 5 to be used as a tool to generate a pulse sequence of two pulses separated by $2 \tau$. This type of phase modulation can be understood in terms of the shifting property of linear phase functions as discussed above. In the upper panel of Fig. 5 half the spectrum (i.e. the low frequency part) is shifted by $t=-\tau$ whereas the other half of the spectrum (i.e. the high frequency part) is shifted to $t=$ $+\tau$. This procedure results in two detuned pulses: an initial red-detuned pre-pulse and a blue-detuned post-pulse. Because both the partial spectra are truncated, the resulting sub-pulses are very long. Shifting the cusp away from the central frequency controls the share for both partial spectra and hence the intensity of the respective components. In the middle panel of Fig. 5 the cusp is shifted towards low frequencies implying a weak red-detuned pre-pulse and an intense blue-detuned post-pulse. When the spectral phase function is mirrored with respect to the central frequency, the temporal envelope is reversed and the temporal phase is conjugated. This operation results in a red-detuned intense pre-pulse and a weak blue-detuned post-pulse.
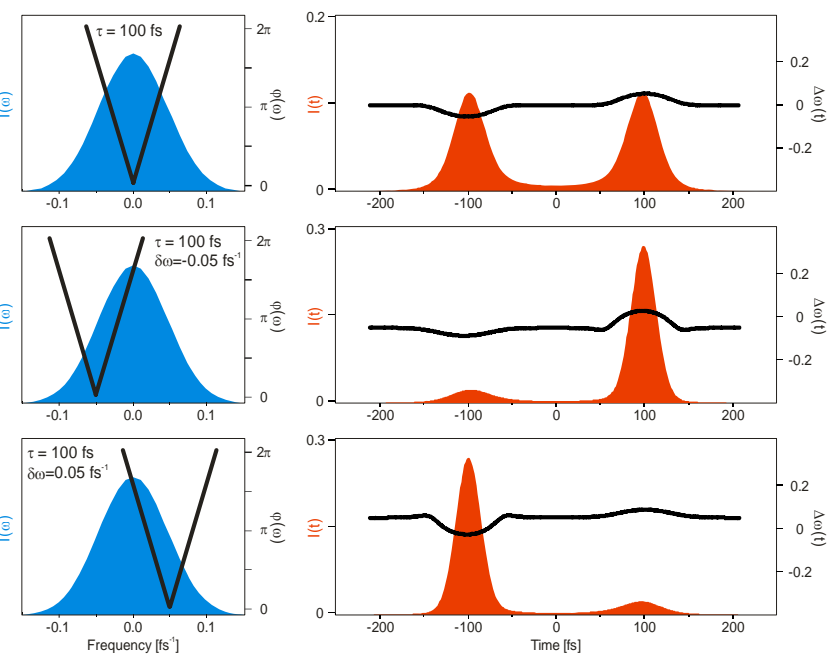

Fig. 5 The function $\varphi(\omega)=\tau|\omega-\delta \omega|$ produces a V-shaped spectral phase. Physically, each portion of the spectrum where the phase is linear corresponds to a (long) pulse shifted by $\pm \tau$. In this way, a sequence of a red-detuned pre-pulse and a blue-detuned postpulse. 


\subsection{Combinations of modulation functions}

Linear combinations of the above phase masks can be used to tailor pulses for specific applications. If a sum of multiple spectral phase functions $\varphi_{i}(\omega)$ is applied the combined action of the linear combination can be decomposed into subsequent execution of the corresponding individual phase functions.

$M(\omega)=e^{-i\left[\varphi_{1}(\omega)+\varphi_{2}(\omega)\right]}=e^{-i \varphi_{1}(\omega)} e^{-i \varphi_{2}(\omega)}=M_{1}(\omega) M_{2}(\omega)$

For example combining sinusoidal phase modulation with quadratic phase function yields a sequence of chirped pulses [33]. In general, the effect of combinations of spectral phase functions on the temporal pulse shape is not always easy to predict due to interference effects among the subsequent modulations.

\section{Material processing with shaped laser pulses}

In this section experimental investigations on the ablation of fused silica by focused unshaped and shaped femtosecond laser radiation are presented. Thereby unshaped (bandwidth limited), positive or negative cubic phaseshaped laser radiation is applied on the surface by single pulses. A short description of the setup is presented here and is also given in $[34,35]$. The resulting structure diameters, detected afterwards by scanning electron microscopy (SEM) and occasionally by atomic force microscopy (AFM), are compared.

\subsection{Experimental setup}

Linear polarized laser radiation with 35 fs full width at half maximum (FWHM) pulse duration and a central wavelength of $790 \mathrm{~nm}$ are provided by an amplified Ti:Sapphire laser system (Femtolasers Femtopower Pro). Temporal shaping of the laser pulse is achieved by the spectral phase modulator (Figure 6). The radiation is focused via a Zeiss Epiplan 50x/0.5NA objective to a lateral spot diameter of $1.4 \mu \mathrm{m}$ resulting from the $1 / \mathrm{e}^{2}$ intensity profile calculation of the point spread function [36]. The diameters of the generated structures for unshaped radiation four times above threshold are consistent with the calculated lateral spot diameter. The calculated axial spot size in air is $9.1 \mu \mathrm{m}$.

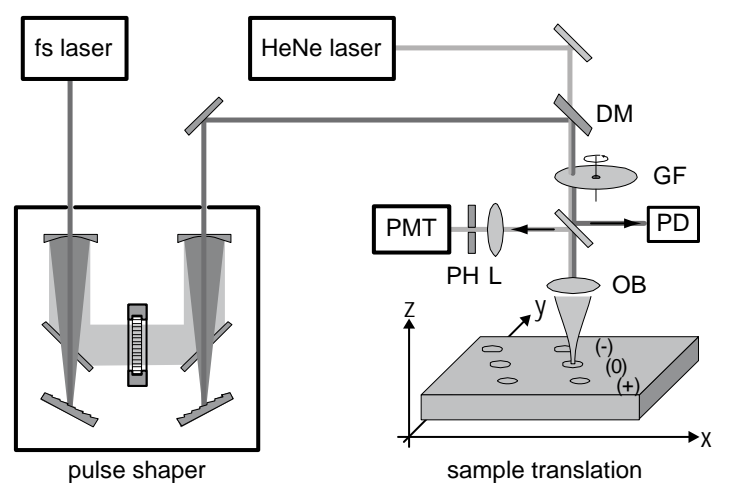

Fig. 6: Experimental setup: The femtosecond laser beam is aligned into the spectral phase modulator. Energy is controlled by a motorized neutral density gradient filter (GF) and monitored with a photodiode (PD). A microscope objective (OB) focuses the beam on the dielectric sample. Confocal surface probing is done by a HeNe laser overlayed by a dielectric mirror (DM), the reflection from the surface is separated by a beamsplitter and guided through a lens $(\mathrm{L})$ and a pinhole $(\mathrm{PH})$ on a photomultiplier tube (PMT). Sample translation is done by a 3 -axis piezo table.

Smallest pulse duration in the interaction region is obtained by adjusting the prism compressor in the fs laser system precompensating the dispersion of the optical elements in the subsequent beam path. Temporal pulse characterization is achieved by $2^{\text {nd }}$ order autocorrelation and crosscorrelation by inserting a Mach-Zehnder interferometer in the beam path and detecting the radiation by a 2-photon photodiode after the objective.

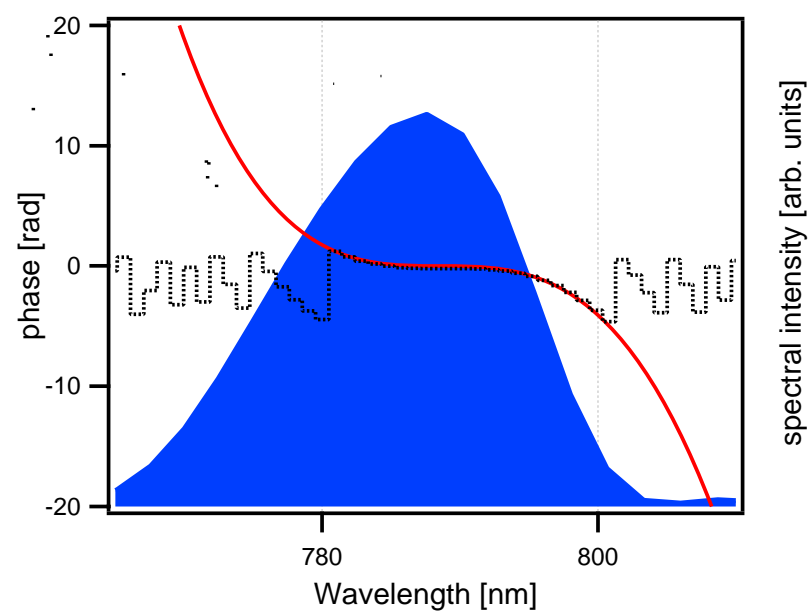

Fig. 7: Blue: spectral intensity of the laser pulse, red solid line shows phase mask for $\varphi_{3}=+6 \times 10^{5} \mathrm{fs}^{3}$, black dashed line shows spectral phase wrapped to an interval of $[0 ; 2 \pi]$ as applied to the LCD.

Applying a cubic phase mask (see Fig. 7) on the phase modulator results in an asymmetric pulse in time domain. The corresponding crosscorrelation between an unshaped and shaped pulse is shown in Fig. 8.

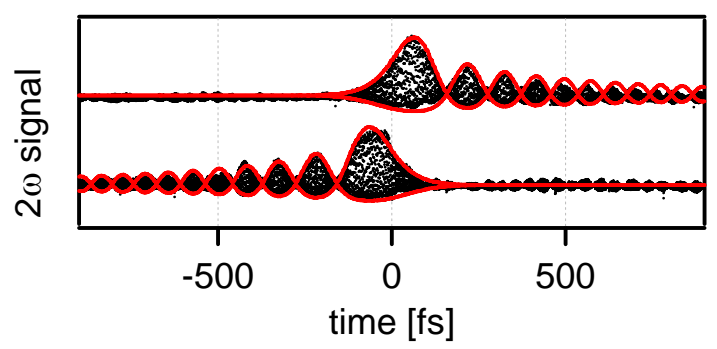

Fig. 8: Simulated (red solid line) and measured (black dots) cross-correlations for a cubic phasemask of $+6 \times 10^{5} \mathrm{fs}^{3}$ (top) and $-6 \times 10^{5} \mathrm{fs}^{3}$ (bottom).

The fused silica sample (Heraeus Suprasil) was cleaned prior and after material processing using an alkaline $(\mathrm{KOH}$ solved in isopropanol) and dilute hydrochloric acid solutions in a supersonic bath. For material processing shaped femtosecond laser pulses are focused onto the sample surface. The single shot pulse energy is adjusted by a motor driven gradient neutral density filter and recorded with a photodiode. The photodiode was calibrated with a commercial powermeter (Ophir Nova 2 with PD300). The sample is translated by a 3-axis piezo table to a new sample position for each shot. Reproducible z-positioning is assured by confocal probing of the sample surface with cw$\mathrm{HeNe}$ laser radiation prior to material processing (see Fig. 6). In a typical measurement pattern (see Fig. 9 and Fig. 
10) the spectral phase mask, energy and focal z-position are varied. Labels and markings are added in the same processing step. The threshold for material damage is determined visually from the scanning electron microscopy (SEM) images (see Fig. 10).

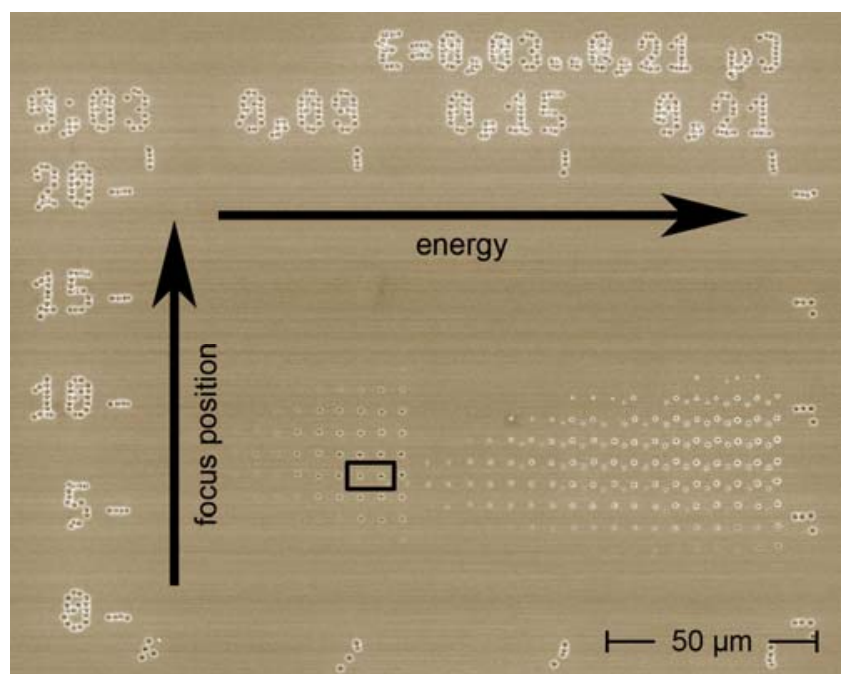

Fig. 9: SEM micrograph of microstructures generated with different parameters regarding focal position, energy and used phase mask. Energy is increased in $6 \mathrm{~nJ}$ steps from left to right. From bottom to top the focal position is changed in steps of $1 \mu \mathrm{m}$ : the focal plane is varied from above the surface to well below the sample surface. The black rectangle marks the enlarged region given in Fig. 10.

\subsection{Results and discussion}

Comparing ablation threshold energies for positive and negative cubic phase parameters one reveals that the threshold is always smaller for positive cubic phases than for negative cubic phases for the same value. As the pulses feature identical fluence, spectrum and pulse duration different asymmetric temporal shape, i. e. the energy distribution in time has a significant influence on the material damage threshold.

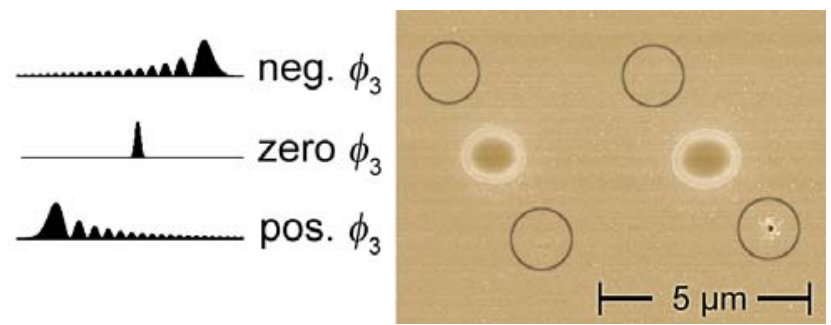

Fig. 10: For each focal position and energy three laser pulses are applied with slightly displaced lateral positions on the substrate (right). Corresponding temporal pulse forms are sketched on the left. On top negative TOD is applied. For the energy used to create the left structure with the unshaped pulse the threshold energy for shaped pulses is not yet reached resulting in not observable structural change (regions are marked with circles). In the middle the unshaped pulses are applied. On the bottom the threshold energy for material processing using positive TOD is reached (detail SEM micrograph for region marked in Fig. 9.).

Additionally the shape and size of the generated structures change from bandwidth limited pulses to pulses with positive cubic phase $\left(+6 \times 10^{5} \mathrm{fs}^{3}\right)$ as shown Fig. 10. Applying laser radiation with a positive cubic phase a reduction up to an order of magnitude in the structure diameter is observed (as small as $100 \mathrm{~nm}$ for radiation with $\varphi_{3}=+6 \times 10^{5} \mathrm{fs}^{3}$ ). For the negative cubic phase $\left(-6 \times 10^{5} \mathrm{fs}^{3}\right)$ the threshold for material processing is larger than for the positive cubic phase, while the structure size is also well below the diffraction limit.

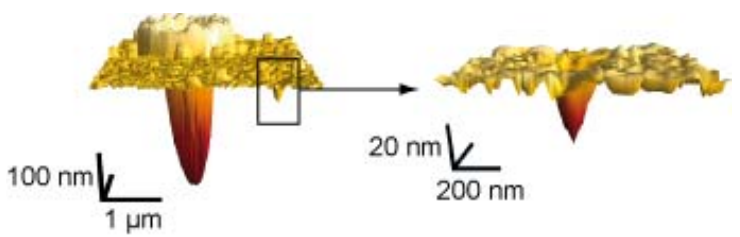

Fig. 11: AFM micrograph of the highlighted area of Fig. 9. Comparison of ablation structures generated by a bandwidth limited pulse together with a structure generated by a temporal shaped pulse $\left(\varphi_{3}=+6 \times 10^{5} \mathrm{fs}^{3}\right)$ at its threshold energy (see Fig. 10).

Occasional crosschecks of topology are performed with an AFM (see Fig. 11) and are in agreement with the SEM data $[34,35]$. However, the structure sizes of both positive and negative $\varphi_{3}$ are significantly smaller than for unshaped pulses at their threshold. In addition, the increase of structure size with increasing pulse energy obtained with cubic phase masks is not as pronounced as with bandwidth limited radiation (see Fig. 13). Applying shaped femtosecond laser radiation using the same optical elements results in the same spot diameter of $1.4 \mu \mathrm{m}$ as applying unshaped radiation. However the sizes of the obtained structures are below $300 \mathrm{~nm}$ over a large energy range thus providing a large process window for generation of nanostructures. The smallest structures are one order of magnitude below the diffraction limit (see Fig. 13). Even with geometrically overlapping focal regions of the consecutively placed laser pulses the nanoscale structures stay separated and exhibit no incubation effects or ripples (see Fig. 12). In contrast to reported structure diameters of below $100 \mathrm{~nm}$ and occasionally up to $15 \mathrm{~nm}$ created at the backside of the substrate using high NA immersion objectives [37] and additionally in conjunction with super resolving pupil plane filters [38] our method works with a long-distance air objective directly on the surface side of the substrate.

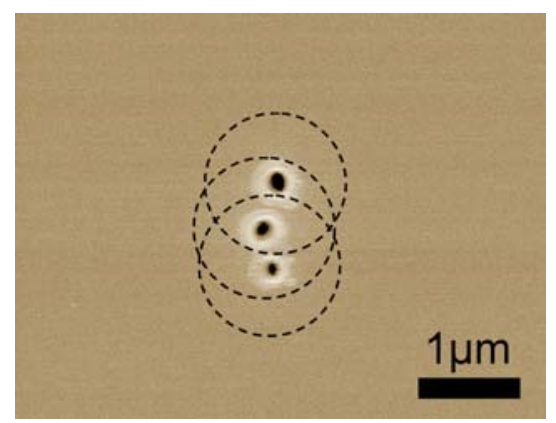

Fig. 12: SEM micrograph of ablation structures in fused silica for temporal shaped $\left(\varphi_{3}=+6 \times 10^{5} \mathrm{fs}^{3}\right)$ laser pulses. The obtained diameters are well below the diffraction limited focus area as marked by dashed circles. 
Material processing depends on reaching a critical electron density mainly generated by two processes: multi photon ionization (MPI) and avalanche ionization (AI). A full kinetic approach was numerically solved in [39] calculating the time dependent electron density using the two ionization processes including free-electron heating via electronphonon-photon collisions and relaxation via electronphonon collisions. To reduce the complexity of this detailed kinetic approach the multiple rate equation (MRE) was introduced, providing a widely applicable model which is valid on a broad range of timescales [40].

MPI provides seed electrons which acquire additional energy via free carrier absorption. A precondition for starting the AI is that these electrons reach the bandgap energy. Using the unshaped and therefore bandwidth limited pulse the dominant process is MPI. For the shaped pulses one has the possibility to control the efficiency of the AI [34,35]. Ultra-fast laser radiation featuring a positive cubic phase mask provides a first strong pulse leading to MPI and thus enabling AI for efficient generation of free electrons.

For the case with negative cubic phase mask the AI does not start till the end of the pulse and therefore the electron generation process is mostly based on MPI leading to a smaller free electron density. Within this description the smaller threshold energy for positive cubic shaped pulses compared to the negative cubic shaped pulses can be explained and is consistent with calculations using the MRE [34].

These experiments with asymmetric ultrashort lasers pulses demonstrate the potential of temporal pulse shaping for high precision micro- and nanomachining.

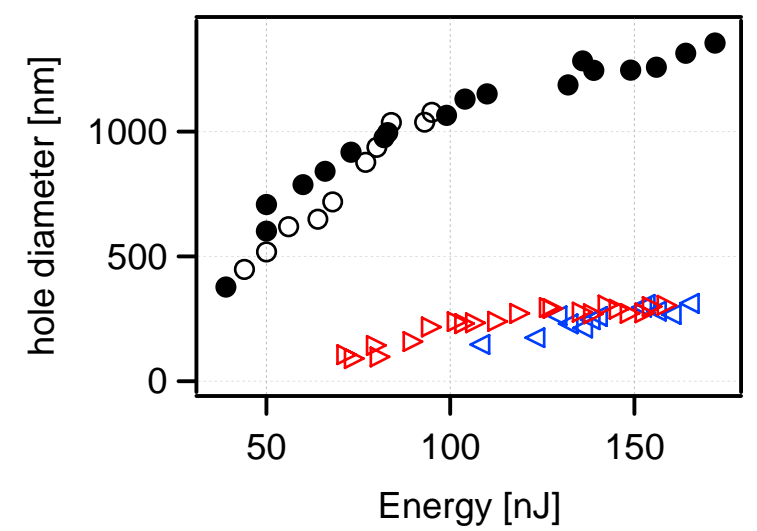

Fig. 13: (Color online) Hole diameters of ablation structures for fused silica with $\varphi_{3}=0 \mathrm{fs}^{3}$ (open and closed circles for two completely independent measurements) for $\varphi_{3}=+6 \times 10^{5} \mathrm{fs}^{3}$ (red triangles) and $\varphi_{3}=-6 \times 10^{5} \mathrm{fs}^{3}$ (blue triangles). Note that the hole diameter obtained with the latter parameters is remarkably robust with respect to variations in laser intensity.

\section{Conclusions}

In this contribution femtosecond pulse shaping techniques with a focus on pulse shapes that might be useful in material processing applications have been highlighted. Pulse shaping via spectral phase modulation has been described generally and has been discussed for polynomial, periodic, step-like or V-shaped spectral phase functions, resulting in shaped laser radiation exhibiting in the temporal regime chirped pulses, or symmetric and asymmetric multi pulses with different temporal phases.

An application to shaped ultra-short laser radiation by spectral phase modulation for micromachining was presented Applying positive and negative cubic spectral phases structures have been generated by ablation of fused silica with hole diameters about $100 \mathrm{~nm}$, being one order of magnitude below the diffraction limited diameter of the focused laser radiation, here $1.4 \mu \mathrm{m}$. We believe that sketched strategies open the route to robust laser processing on the nanometer scale.

\section{Acknowledgments and Appendixes}

The authors wish to acknowledge the support of DFG.

\section{References}

[1] L. Sudrie, A. Couairon, M. Franco, B. Lamouroux, B. Prade, S. Tzortzakis, and A. Mysyrowicz: Phys.Rev.Lett. 89, (2002) 186601.

[2] A. Vogel and V. Venugopalan: Chem.Rev. 103, (2003) 577.

[3] F. Dausinger, F. Lichtner, and H. Lubatschowski: "Femtosecond Technology for Technical and Medical Applications" ed. by F. Dausinger, F. Lichtner, and H. Lubatschowski (Springer, Berlin Heidelberg, 2004).

[4] S. S. Mao, F. Quéré, S. Guizard, X. Mao, R. E. Russo, G. Petite, and P. Martin: Appl.Phys.A 79, (2004) 1695.

[5] A. Vogel, J. Noack, G. Hüttman, and G. Paltauf: Appl.Phys.B 81, (2005) 1015.

[6] H. Misawa, and S. Juodkazis: "3 D Laser Microfabrication" ed. by Misawa, H. and Juodkazis, S. (WILEY-VCH Verlag GmbH \& Co. KGaA, 2006).

[7] B. C. Stuart, M. D. Feit, A. M. Rubenchik, B. W. Shore, and M. D. Perry: Phys.Rev.Lett. 74, (1995) 2248.

[8] M. Lenzner, J. Krüger, S. Sartania, Z. Cheng, Ch. Spielmann, G. Mourou, W. Kautek, and F. Krausz: Phys.Rev.Lett. 80, (1998) 4076.

[9] A. C. Tien, S. Backus, H. C. Kapteyn, M. M. Murnane, and G. Mourou: Phys.Rev.Lett. 82, (1999) 3883

[10] M. Li, S. Menon, P. Nibarger, and G. N. Gibson: Phys.Rev.Lett. 82, (1999) 2394.

[11] G. Petite, S. Guizard, P. Martin, and F. Quéré: Phys.Rev.Lett. 83, (1999) 5182.

[12] Y. P. Deng, X. H. Xie, H. Xiong, Y. X. Leng, C. F. Cheng, H. H. Lu, R. X. Li, and Z. Z. Xu: Optics Express 13, (2005) 3096.

[13] L. Englert, M. Wollenhaupt, B. Rethfeld, L. Haag, C. Sarpe-Tudoran, and T. Baumert: Proceeding of the Fourth International WLT-Conference on Lasers in Manufacturing 2007 (2007)

[14] R. Stoian, M. Boyle, A. Thoss, A. Rosenfeld, G. Korn, and I. V. Hertel: Appl.Phys.A 77, (2003) 265.

[15] M. Wollenhaupt, V. Engel, and T. Baumert: Ann.Rev.Phys.Chem. 56, (2005) 25.

[16] Brixner, T., Pfeifer, T., Gerber, G., Wollenhaupt, M., and Baumert, T.: "Femtosecond Laser Spectroscopy" ed. by Hannaford, P. (Springer Verlag, 2005). 
[17] M. Wollenhaupt, D. Liese, A. Präkelt, C. SarpeTudoran, and T. Baumert: Chem.Phys.Lett. 419, (2006) 184.

[18] V. V. Lozovoy, X. Zhu, T. C. Gunaratne, D. A. Harris, J. C. Shane, and M. Dantus: J.Phys.Chem.A 112, (2008) 3789.

[19] A. Präkelt, M. Wollenhaupt, A. Assion, C. Horn, C. Sarpe-Tudoran, M. Winter, and T. Baumert: Rev.Sci.Instr. 74, (2003) 4950.

[20] A. M. Weiner: Rev.Sci.Instr. 71, (2000) 1929.

[21] M. Wollenhaupt, A. Assion, and T. Baumert: "Springer Handbook of Lasers and Optics" ed. by F. Träger (Springer Science and Business Media, 2007).

[22] A. Präkelt, M. Wollenhaupt, C. Sarpe-Tudoran, and T. Baumert: Phys.Rev.A 70, (2004) 063407.

[23] M. Wollenhaupt, A. Präkelt, C. Sarpe-Tudoran, D. Liese, and T. Baumert: Appl.Phys.B 82, (2006) 183.

[24] J. D. McMullen: JOSA 67, (1977) 1575.

[25] M. Wollenhaupt, A. Assion, O. Bazhan, C. Horn, D. Liese, C. Sarpe-Tudoran, M. Winter, and T. Baumert: Phys.Rev.A 68, (2003) 015401.

[26] M. Wollenhaupt, A. Präkelt, C. Sarpe-Tudoran, D. Liese, and T. Baumert: J.Opt.B 7, (2005) S270.

[27] M. Renard, R. Chaux, B. Lavorel, and O. Faucher: Optics Express 12, (2004) 473.

[28] M. Wollenhaupt, A. Präkelt, C. Sarpe-Tudoran, D. Liese, T. Bayer, and T. Baumert: Phys.Rev.A 73, (2006) 063409.

[29] D. Meshulach and Y. Silberberg: Nature 396, (1998) 239.

[30] D. Meshulach and Y. Silberberg: Phys.Rev.A 60, (1999) 1287.

[31] P. Panek and A. Becker: Phys.Rev.A 74, (2006) 023408.

[32] T. Bayer, M. Wollenhaupt, C. Sarpe-Tudoran, and T. Baumert: Phys.Rev.Lett. 102, (2009) 023004-1.

[33] T. Bayer, M. Wollenhaupt, and T. Baumert: J.Phys.B 41, (2008) 074007.

[34] L. Englert, B. Rethfeld, L. Haag, M. Wollenhaupt, C. Sarpe-Tudoran, and T. Baumert: Optics Express 15, (2007) 17855.

[35] L. Englert, M. Wollenhaupt, L. Haag, C. SarpeTudoran, B. Rethfeld, and T. Baumert: Appl. Phys. A 92, (2008) 749.

[36] M. Martinez-Corral: Wave-Optical Systems Engineering II, Proceedings of SPIE Vol 5182, 112-122 (2003).

[37] A. P. Joglekar, H. Liu, G. J. Spooner, E. Meyhöfer, G. Mourou, and A. J. Hunt: Appl.Phys.B 77, (2003) 25.

[38] M. Merano, G. Boyer, A. Trisorio, G. Chériaux, and G. Mourou: Opt.Lett. 32, (2007) 2239.

[39] A. Kaiser, B. Rethfeld, M. Vicanek, and G. Simon: Phys.Rev.B 61, (2000) 11437.

[40] B. Rethfeld: Phys.Rev.Lett. 92, (2004) 187401-1.

(Received: June 16, 2008, Accepted: September 24, 2009) 